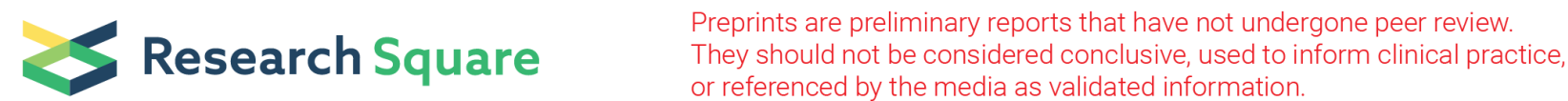

\section{Effects of polygenic risk score of type 2 diabetes on the hippocampal topological property and episodic memory}

\section{Yang Zhang}

Tianjin Medical University General Hospital

\section{Xin Du}

Tianjin Medical University General Hospital

\section{Yumeng Fu}

Tianjin Medical University General Hospital

Qiuyue Zhao

Tianjin Medical University General Hospital

\section{Zirui Wang}

Tianjin Medical University General Hospital

\section{Wen Qin}

Tianjin Medical University General Hospital

Quan Zhang ( $\nabla$ quanzhang@tmu.edu.cn )

Tianjin Medical University General Hospital https://orcid.org/0000-0002-9776-2401

\section{Research Article}

Keywords: Type 2 diabetes, polygenic risk score, hippocampus, topological network, episodic memory

Posted Date: December 3rd, 2021

DOI: https://doi.org/10.21203/rs.3.rs-1102711/v1

License: (c) (i) This work is licensed under a Creative Commons Attribution 4.0 International License. Read Full License 


\section{Abstract \\ Purpose}

Type 2 diabetes is associated with a higher risk of dementia. The pathogenesis is complex, partly influenced by genetic factors. The hippocampus is the most vulnerable brain region in individuals with type 2 diabetes. However, whether the genetic risk of type 2 diabetes is associated with the hippocampus and episodic memory remains unclear. This study explored the influence of polygenic risk score (PRS) of type 2 diabetes on the white matter topological properties of the hippocampus among individuals with and without type 2 diabetes and its associations with episodic memory.

\section{Methods}

This study included 103 individuals with type 2 diabetes and 114 well-matched individuals without type 2 diabetes. All the participants were genotyped, and a diffusion tensor imaging-based structural network was constructed. PRS was calculated based on a genome-wide association study of type 2 diabetes. The PRS-by-disease interactions on the bilateral hippocampal topological network properties were evaluated by analysis of variance (ANOVA).

\section{Results}

There were significant PRS-by-disease interaction effects on the nodal topological properties of the right hippocampus node. In the individuals with type 2 diabetes, the PRS was correlated with the right hippocampal nodal properties, and the nodal properties were correlated with the episodic memory. In addition, the right hippocampal nodal properties mediated the effect of PRS on the episodic memory in individuals with type 2 diabetes.

\section{Conclusion}

Our results suggested a gene-brain-cognition biological pathway, which might help understand the neural mechanism of the genetic risk of type 2 diabetes affects episodic memory in type 2 diabetes.

\section{Introduction}

Type 2 diabetes is associated with accelerated brain aging and a higher risk of dementia in late life(Riederer et al., 2017). However, the underlying neural mechanisms responsible for cognitive decline in type 2 diabetes have not been fully elucidated. The pathogenesis of diabetes is complex. A better understanding of the factors associated with cognitive decline, including brain and genetic markers, will facilitate early clinical intervention to attenuate severe cognitive dysfunction or dementia. 
The hippocampus is the most vulnerable brain region in individuals with type 2 diabetes (Sanjari Moghaddam, Ghazi Sherbaf, \& Aarabi, 2019), and episodic memory impairment related to the hippocampus is the most prominent and specifically affected cognitive domain in individuals with type 2 diabetes (McCrimmon, Ryan, \& Frier, 2012). Hippocampus atrophy has been frequently reported in individuals with type 2 diabetes with cognitive decline(Hempel, Onopa, \& Convit, 2012; Milne et al., 2018; Zhang et al., 2014). Studies showed weaker functional connectivities between the hippocampus and other brain regions in individuals with type 2 diabetes, correlated with memory performance(Sun et al., 2018; Zhou et al., 2010). In recent years, several diffusion tensor imaging (DTI) studies identified white matter (WM) alterations in the hippocampus and hippocampus-related WM fibers in individuals with type 2 diabetes (Qi et al., 2017; Sanjari Moghaddam et al., 2019). Of significant importance, by using a graphtheoretical approach (Xiong et al., 2020), the hippocampal topological properties have been found altered and correlated with cognitive impairment in individuals with type 2 diabetes, suggesting that DTI topological network properties could be a sensitive marker of cognitive decline even when there is no sign of macrostructural gray matter (GM) volume abnormalities and atrophy of brain structures(Sanjari Moghaddam et al., 2019).

There is ample evidence that the individual risk of type 2 diabetes is strongly influenced by genetic factors, with a heritability of $26 \%-69 \%$ depending on the age of onset(Padilla-Martínez, Collin, Kwasniewski, \& Kretowski, 2020). Over the past decades, more than a hundred genetic loci associated with type 2 diabetes risk have been discovered using genome-wide association studies (GWAS) (Spracklen et al., 2020). Although these variants have only a low or moderate impact on type 2 diabetes' disease risk individually, combining multiple loci into a polygenic score may have significant predictive utility and potentially influence clinical management. Here we introduced a polygenic risk score (PRS), which accumulates the total risk of a phenotype in an individual based on variants present in their genome(Padilla-Martínez et al., 2020). In some cognitive changes-related diseases such as AD(de Rojas et al., 2021) and schizophrenia(Wimberley et al., 2017), the PRS of the disease has been proven to predict correlates of the disease risk and related brain structural perturbations. However, whether and how the type 2 diabetes' PRS is related to the brain abnormality has not been documented.

In the current study, we examined the influence of type 2 diabetes-related PRS on the WM topological network properties of the hippocampus among individuals with an without type 2 diabetes, and its associations with episodic memory function. Genetic risk for type 2 diabetes was determined using a PRS that integrated 97 type 2 diabetes risk single-nucleotide polymorphisms (SNPs). Because the hippocampus is the most vulnerable target in type 2 diabetes, we focused our analyses on the hippocampus and its relationship with the episodic memory performance. Thus, we hypothesized that the PRS would affect the hippocampal topological network properties of the individuals with type 2 diabetes and that the WM topological network properties of the hippocampus would mediate the effect of the PRS on episodic memory.

\section{Method}




\section{Participant}

The individuals with and without type 2 diabetes in this study were recruited from the endocrinology department of Tianjin Medical University General Hospital and from community recruitment respectively between 2018 and 2019. A total of 109 individuals with type 2 diabetes and 119 individuals without type 2 diabetes were initially recruited. Four individuals with type 2 diabetes and five individuals without type 2 diabetes were excluded due to poor blood sample quality. Two individuals with type 2 diabetes were excluded due to poor image quality. Finally, 103 individuals with type 2 diabetes and 114 wellmatched individuals without type 2 diabetes were enrolled. Type 2 diabetes were diagnosed according to the 2010 criteria of the American Diabetes Association (ADA)(Association, 2010). Individuals with type 2 diabetes related complications, including peripheral neuropathy, retinopathy, and nephropathy were excluded from the study. None of the individuals had experienced severe hypoglycemia during the past two years. All the participants were right-handed. Exclusion criteria were: (1) previous history of brain disease, including stroke, epilepsy, trauma or hemorrhage; (2) Mini-Mental State Examination (MMSE) (Folstein, Folstein, \& McHugh, 1975) score < 27; (3) psychiatric or neurologic disorders that may affect cognition; (4) alcohol or drug abuse; and (5) contraindications for MRI scan. All subjects underwent a series of standardized clinical evaluations.

All the participants were in Chinese Hans. Height, weight, and body mass index (BMI) were measured for each participant. The criteria for hypertension were systolic blood pressure $>140 \mathrm{mmHg}$ or diastolic blood pressure $>90 \mathrm{mmHg}$ or taking antihypertensive medications. Fasting blood glucose (FBG) and glycosylated hemoglobin (HbA1c) were measured via standard laboratory testing.

\section{Neuropsychological testing}

A battery of cognitive assessments was performed before MRI. General cognition was assessed with the MMSE(Folstein et al., 1975). Anxiety and depression were evaluated with the Self-Rating Anxiety Scale(Zung, 1971) and the Self-Rating Depressive Scale(Zung, 1965), respectively. Episodic memory was assessed by two tests: (1) The Rey-Osterrieth Complex Figure Test (ROCF)(Shin, Park, Park, Seol, \& Kwon, 2006) which consists of three conditions: copy, immediate recall, and delayed recall. (2) The Chinese version of the Auditory Verbal Learning Test (AVLT)(Rosenberg, Ryan, \& Prifitera, 1984) including shortand long-term memory.

\section{Image data acquisition}

All imaging data were obtained on a 3.0-T MR system (Discovery MR750; General Electric, Milwaukee, WI, USA), equipped with an 8-channel phase-array head coil. DTI images were acquired by a single-shot echo planar imaging $(\mathrm{EPI})$ sequence with the following parameters: repetition time $(\mathrm{TR})=7000 \mathrm{~ms}$; echo time $(T E)=95 \mathrm{~ms}$; flip angle $(F A)=12^{\circ} ;$ field of view $(F O V)=256 \mathrm{~mm} \times 256 \mathrm{~mm}$; matrix $=128 \times 128$; slice thickness $=3 \mathrm{~mm}$, no gap; 48 axial slices; 64 encoding diffusion directions with $b=1,000 \mathrm{~s} / \mathrm{mm}^{2}$, and 12 non-diffusion $b=0 \mathrm{~s} / \mathrm{mm}^{2}$ images. The Sagittal T1-weighted images were obtained using a brain volume 
sequence, as follows: $\mathrm{TR}=8.2 \mathrm{~ms}, \mathrm{TE}=3.2 \mathrm{~ms}, \mathrm{TI}=450 \mathrm{~ms}, \mathrm{FA}=12^{\circ}$, matrix $=256 \times 256$, and 188 continued sagittal sections with section thickness of $1 \mathrm{~mm}$.

\section{Imaging preprocess and network construction}

The DTI data preprocessing steps were performed using a PANDA toolbox(Cui, Zhong, Xu, He, \& Gong, 2013) based on FMRIB Software Library v5.0(Jenkinson, Beckmann, Behrens, Woolrich, \& Smith, 2012), briefly including brain extraction, realignment, eddy current and motion artifact correction, fractional anisotropy calculation, and diffusion tensor tractography. An FA threshold of 0.2 and a turning angle threshold of $45^{\circ}$ in the Fiber Assignment by Continuous Tracking (FACT) algorithm were set when tracking WM fibers.

An Automated Anatomical Labeling (AAL) atlas was used to parcellate each brain into 90 anatomical regions. For detailed parcellation process please see the Online Resource. Each AAL region was taken as a node, and interconnections between brain regions were taken as the edges of the structural network. Interconnections between brain regions were considered present if at least three WM fibers were present between the regions, as in previous studies(Shu et al., 2012). The WM fiber number (FN) threshold was set as three. Finally, a binary $90 \times 90$ matrix for each subject was obtained.

The nodal topological properties including the nodal global efficiency (NGe), nodal local efficiency (NLe), nodal clustering coefficient (NCp), nodal shortest path length (NLp), and nodal degree (Nd) were calculated with the graph-theoretical network analysis toolbox (GRETNA; http://www.nitrc.org/projects/gretna). The explanation of these properties was documented in the Online Resource. Detailed calculation methods have been documented in a previous article(Rubinov \& Sporns, 2010).

\section{Genotyping}

All the participants were genotyped using the Illumina Infinium Asian Screening Array (ASA) (https://support.illumina.com.cn/array/array_kits/infinium-asian-global-screening-array.html), a highthroughput genotyping chip designed for the Asian population (Illumina Asian screening array chip) with 700,000 sampling SNPs.

\section{Polygenic risk score}

After the strict QC and imputation process (for detailed steps please see Online Resource), PRS was calculated. A recent meta-analyzed GWAS of type 2 diabetes in East Asian individuals (including 77418 individuals with type 2 diabetes and 356,122 individuals without type 2 diabetes)(Spracklen et al., 2020) has identified 111 type 2 diabetes risk SNPs (adjusted for BMI), 97 of which were found in our imputed genotype dataset. The 97 risk SNPs were used for the calculation of PRS. The score was calculated on SNPs reaching genome-wide significance $\left(P=5 \times 10^{-8}\right)$. For each participant, the PRS was 
calculated by multiplying the number of risk alleles for each SNP by the weight for that SNP, and then taking the sum across the 97 SNPs, according to the following formula(De Jager et al., 2009):

$$
\begin{gathered}
\omega_{O R i}=\ln (O R) \\
G R S=\sum_{i=1}^{I} \omega_{O R i} G_{i}
\end{gathered}
$$

where i was the SNP, was the weight for SNP $i$, and $G_{i}$ was the number of risk alleles. The weight was the natural log of the odds ratio (OR) value of each SNP, which was obtained from the above-mentioned type 2 diabetes GWAS research(Spracklen et al., 2020).

\section{Statistical approach}

\section{Demographic, clinical, behavioral data, and PRS}

Statistical analyses for demographic, clinical and behavioral data were performed using Statistical Package for Social Sciences (SPSS, v. 23.0, IBM SPSS Statistics, IBM Corporation). The two samples Ttest was used for continuous variables, and the Chi-square $(\chi 2)$ test was used for categorical variables. The significant level was set at $P<0.05$.

\section{PRS-by-disease interaction analysis}

The PRS-by-disease interactions on the bilateral hippocampal topological properties (NGe, NLe, NCp, NLp and $\mathrm{Nd}$ ) were evaluated by analysis of variance (ANOVA) with the hippocampal topological property was treated as a dependent variable, diagnosis (individuals with type 2 diabetes vs. without type 2 diabetes), PRS, and their interaction as interesting independent variables, and age, gender, education years, and BMI as confounding variables. When ANOVA showed a statistically significant interaction effect after the Bonferroni correction, the post hoc correlation analyses were performed to test the relationship between the hippocampal topological properties and the PRS in the individuals with and without type 2 diabetes respectively.

\section{Correlation analysis between hippocampal topological properties and episodic memory}

Partial correlation analyses were performed to test the correlation between the hippocampal topological properties and episodic memory tests after controlling for the age, gender, educational level, and BMI in the individuals with type 2 diabetes and the individuals without type 2 diabetes. Statistical significance was set at $\boldsymbol{P}<0.05$.

\section{Mediation Analysis}

The PROCESS macro version 3.4 implemented in IBM SPSS was adopted to perform the mediation analyses to examine whether the hippocampal topological properties mediated the association between 
the PRS and the episodic memory, with the PRS as the independent variable, the hippocampal topological property as the mediation variable, and the episodic memory as the dependent variables. The age, gender, education years, and BMI were controlled. During the mediation analysis, ordinary least squares regression was used to calculate statistics for specific paths, and 5000 bias-corrected bootstrap resamples were conducted to generate a $\mathrm{Cl}$ for the mediation effect(Preacher \& Hayes, 2008). When the $95 \% \mathrm{Cl}$ does not contain zero, it is considered a significant mediation effect, whereas the main effect for mediation analysis is unnecessary.

\section{Results}

\section{Demographic, clinical and cognitive characteristics}

Participant demographic, clinical and cognitive characteristics are shown in Table 1. The individuals with type 2 diabetes and the individuals without type 2 diabetes were matched for age, gender and education. The weight, BMI, FBG, and $\mathrm{HbA} 1 \mathrm{c}$ were significantly higher in the individuals with type 2 diabetes than the individuals without type 2 diabetes $(P<0.05)$. The ROCF delayed recall score, the AVLT short- and longterm memory scores were significantly lower in individuals with type 2 diabetes than the individuals without type 2 diabetes $(P<0.05)$. 
Table 1

Demographics, Clinical Data, Neuropsychological and Episodic Memory Assessment

Characteristics
Type 2 Diabetes

Diabetes

$(n=103)$

$(n=114)$

\section{Demographics}

Age (years)

Gender (Man/Woman)

Education (years)

Clinical Data

Weight (kg)

Height (cm)

BMI $\left(\mathrm{kg} \mathrm{m}^{-2}\right)$

BP (Hypertension/Normotension)

FBG $\left(\mathrm{mmol} \mathrm{L}^{-1}\right)$

$\mathrm{HbA1c}(\%)$

$\mathrm{HbA1c}\left(\mathrm{mmol} \mathrm{L}^{-1}\right)$

Neuropsychological Test

MMSE

SAS

SDS

Episodic Memory

ROCF Immediate Recall

ROCF Delayed Recall

AVLT Short-term Memory

AVLT Long-term Memory
$60.15 \pm 6.72$

$54 / 49$

$10.87 \pm 2.52$

$72.94 \pm 10.94$

$167.02 \pm 8.25$

$26.03 \pm 2.70$

$53 / 50$

$9.25 \pm 3.63$

$7.29 \pm 1.43$

$56.15 \pm 15.61$

$27.95 \pm 1.67$

$36.80 \pm 9.11$

$37.83 \pm 10.75$

$37.25 \pm 8.34$

$27.88 \pm 1.86$

$0.293^{a}$

0.770

$35.37 \pm 7.28$

$1.281^{\mathrm{a}}$

$0.447^{a}$

0.655
0.105

0.382

0.975

$<0.001^{*}$

0.255

$<0.001^{*}$

0.137

$<0.001^{\star}$

$<0.001^{\star}$

$<0.001^{\star}$

$11.455^{\mathrm{a}}<0.001^{\star}$

0.202

0.071

$0.007^{\star}$

$16.64 \pm 6.33 \quad 19.00 \pm 6.47 \quad-2.703^{\mathrm{a}}$

0.038 *

$41.39 \pm 8.36$

$44.00 \pm 9.94$

$-2.083^{a}$

$0.012^{\star}$ 
Diabetes

$$
(n=103) \quad(n=114)
$$

*Significant level $\mathrm{P}<0.05^{\text {a }}$ presents $T$ values, ${ }^{\mathrm{b}}$ presents $\chi 2$ values. Values are mean \pm standard deviation or number of subjects. AVLT, Auditory Verbal Learning Test; FBG, fasting blood glucose; MMSE, mini-mental state examination; ROCF, Rey-Osterrieth Complex Figure Test; SAS, self-rating anxiety scale; SDS, self-rating depression scale.

\section{PRS-by-disease interaction on WM topological network properties of the hippocampus}

There were significant PRS-by-disease interaction effects on three topological properties of the right hippocampus after multiple comparison correction, including the NGe $(F=11.868, \mathrm{P}<0.001)$, NLp $(F=$ 11.497, $\mathrm{P}<0.001)$ and $\mathrm{Nd}(F=11.547, \mathrm{P}<0.001)$. Detailed statistic results were listed in Table 2 . The post hoc analyses identified significant correlation between the PRS and the three right hippocampal nodal properties in the individuals with type 2 diabetes (NGe: $r=0.366, \mathrm{P}<0.001$; NLp: $r=-0.366, \mathrm{P}<$ $0.001 ; \mathrm{Nd}: r=0.320, \mathrm{P}<0.001)$, while no such correlation in the individuals with type 2 diabetes. Please see Figure 1. 
Table 2

PRS-by-disease interactions on the bilateral hippocampal nodal properties (FN3)

\begin{tabular}{|c|c|c|c|c|c|c|}
\hline \multirow[t]{3}{*}{ Nodal properties } & \multirow{2}{*}{\multicolumn{2}{|c|}{$\begin{array}{l}\text { Main Effect } \\
\text { (disease) }\end{array}$}} & \multirow{2}{*}{\multicolumn{2}{|c|}{$\begin{array}{l}\text { Main Effect } \\
\text { (PRS) }\end{array}$}} & \multirow{2}{*}{\multicolumn{2}{|c|}{$\begin{array}{l}\text { Interaction Effect } \\
\text { (PRS } ₫ \text { disease) }\end{array}$}} \\
\hline & & & & & & \\
\hline & $\mathbf{F}$ & $\mathbf{P}$ & $\mathbf{F}$ & $\mathbf{P}$ & $\mathbf{F}$ & $\mathbf{P}$ \\
\hline \multicolumn{7}{|l|}{ Left Hippocampus } \\
\hline NGe & 0.353 & 0.553 & 1.16 & 0.283 & 0.133 & 0.716 \\
\hline NLe & 0.787 & 0.376 & 2.419 & 0.121 & 0.021 & 0.884 \\
\hline NCp & 0.018 & 0.895 & 1.998 & 0.159 & 4.487 & 0.998 \\
\hline NLp & 0.351 & 0.554 & 1.888 & 0.171 & 0.249 & 0.618 \\
\hline Nd & 0.722 & 0.396 & 0.489 & 0.485 & 0.327 & 0.568 \\
\hline \multicolumn{7}{|c|}{ Right Hippocampus } \\
\hline NGe & 0.384 & 0.536 & 6.354 & 0.012 & 11.868 & $<0.001 *$ \\
\hline NLe & 0.028 & 0.866 & 2.529 & 0.113 & 0.112 & 0.738 \\
\hline NCp & 0.160 & 0.689 & 3.702 & 0.056 & 1.011 & 0.316 \\
\hline NLp & 0.581 & 0.447 & 7.145 & 0.008 & 11.497 & $<0.001 *$ \\
\hline Nd & 0.299 & 0.585 & 2.126 & 0.146 & 11.547 & $<0.001 *$ \\
\hline
\end{tabular}

\section{Correlation Analysis}

In the individuals with type 2 diabetes, the PRS were positively correlated with the NGe and the Nd, while negatively correlated with the NLp, of the right hippocampus (Fig. 1). Additionally, the NGe of the right hippocampus were positively correlated with three episodic memory test performances, including the ROCF immediate recall score, the ROCF delayed recall score, and the long-term memory score. The NLp of the right hippocampus were negatively correlated with the ROCF immediate recall score, the ROCF delayed recall score, and the long-term memory score. No correlation was found in the individuals without type 2 diabetes. Please see Fig. 2.

\section{Mediation Analysis}


In the individuals with type 2 diabetes, significant indirect effects were found from the PRS to episodic memory mediated by the NGe/ NLp of the right hippocampus. There was no significant direct effect between the PRS and hippocampal nodal property or indirect effect from the PRS to episodic memory performance in the individuals without type 2 diabetes. For details, please see Fig. 3-4.

\section{Discussion}

In this study, there were three main findings. First, significant PRS-by-disease interaction effects on three topological network properties of the right hippocampus were found. The individuals with type 2 diabetes with higher PRS showed higher NGe and Nd, while lower NLp of the right hippocampus. Second, the NGe of the right hippocampus was positively correlated with episodic memory, while the NLp of the right hippocampus was negatively correlated with episodic memory performance. Finally, in the individuals with type 2 diabetes, significant indirect effects from the PRS to episodic memory mediated by the NGe and NLp of the right hippocampus were also found.

The hippocampi are critical brain regions involved in cognitive functions such as learning and memory. Previous studies have identified hippocampi as hub nodes in normal human brain networks, with both ascending and descending pathways responsible for transmitting and modulating memory signals (Li et al., 2020). Normally, the NGe and NLp measure the global information transmission capacity of the network(Rubinov \& Sporns, 2010). We observed a combination of higher NGe and lower NLp in individuals with type 2 diabetes with higher PRS, suggesting that the brains are better organized in those individuals with a higher genetic risk of type 2 diabetes. These results may seem contradictory in view of the known negative impact of type 2 diabetes on cognition. However, a better organized cerebral network in the high PRS individuals can be attributed to an earlier stage of brain damage, where compensatory mechanisms, such as the reorganization of networks, might play a role. A similar brain network compensation phenomenon has been reported in previous graph theoretical network studies on individuals with type 2 diabetes (van Bussel et al., 2016; Xiong et al., 2020). The results of correlations between the nodal network properties and episodic memory also strengthen the explanation of an underlying compensatory mechanism. The individuals with type 2 diabetes in this study did not have clinical complications, and they could still maintain a normal level of general cognition (MMSE) by reorganizing the brain network. However, higher-level cognition tests such as episodic memory declined. Moreover, the patients exhibited a significant correlation between the hippocampal topological properties and episodic memory performances. It suggested that after compensatory brain network reorganization, individuals with higher hippocampal NGe and lower NLp would perform better episodic memory. However, despite such compensation, the ability might be limited. The overall performance of episodic memory in the individuals with type 2 diabetes was still worse than that of the individuals without type 2 diabetes. We speculated that the hippocampal node would gradually decompensate as the disease progressed, and cognitive function would decline significantly. On the contrary, no correlation between hippocampal nodal properties and cognitive performance was found among the individuals without type 2 diabetes, suggesting no such compensation happened. 
Here we noticed that interactive effect was only found in the right hippocampus, suggesting that the PRS had more pronounced effects on the right hippocampus of the patients. In previous studies, hippocampal asymmetry has been discovered in all kinds of individuals, while with controversial findings. The current consensus view is that people with normal cognition usually show that the right hippocampus is larger than the left hippocampus(Pedraza, Bowers, \& Gilmore, 2004). Recently, a topological network analysis of Li et al.(Li et al., 2020) found that only the right hippocampus became the brain hub node, but not the left. And the hub node of the right hippocampus was lost in individuals with type 2 diabetes. These combing results provide a possibility that the right hippocampus is more likely to play a stronger role in early compensation than the left hippocampus.

The mediation analysis further revealed that right hippocampal nodal properties mediated the effect of PRS on episodic memory in individuals with type 2 diabetes. These results provided a gene-braincognition pathway that helps understand how the PRS affects episodic memory in individuals with type 2 diabetes.

Some limitations need to be addressed. First, this study is a cross-sectional study. Thus dynamic changes cannot be obtained. The current results are only applicable to individuals in a specific stage of the disease. Longitudinal studies are still needed to clarify whether these results could be extended to a wider range of patients. Second, because the individuals with type 2 diabetes enrolled in this study need long-term medication to control blood glucose, the effect of treatments cannot be avoided.

In summary, this study verified that the PRS affected the structural topological properties of the right hippocampus node in the individuals with type 2 diabetes, and the nodal property changes of the right hippocampus mediated the effect of PRS on episodic memory. These results suggested a gene-braincognition biological pathway, which might help understand the neural mechanism of the genetic risk of type 2 diabetes affects episodic memory in individuals with type 2 diabetes.

\section{Statements And Declarations}

\section{Funding}

This work was supported by the Natural Science Foundation of Tianjin, China (17JCZDJC36300).

\section{Conflicts of interest}

No potential conflicts of interest relevant to this article were reported.

\section{Availability of data and material}

The data sets generated during and/or analyzed during the current study are not publicly available because they are under construction but are available from the corresponding author upon reasonable request. No applicable resources were generated or analyzed during the current study. 
Acknowledgments

The authors thank all of the volunteers and participants for participating in the study.

\section{Authors' contributions}

Y.Z. and Q.Z. designed research; Y.Z., X.D., Y.F., Q.Z., Z.W., W.Q. and Q.Z. performed research; Y.Z., X.D., W.Q. and Q.Z. analyzed data; Y.Z. and Q.Z wrote the paper. Q.Z. is the guarantor of this work and, as such, had full access to all the data in the study and takes responsibility for the integrity of the data and the accuracy of the data analysis.

\section{Ethics approval and consent to participate}

The study protocol was approved by the Ethics Committee of Tianjin Medical University General Hospital, with written informed consent from all subjects.

\section{Consent for publication}

Not applicable

\section{References}

1. Association, A. D. (2010). Diagnosis and classification of diabetes mellitus. Diabetes Care, 33 Supp/ 1, S62-69. doi:10.2337/dc10-S062

2. Cui, Z., Zhong, S., Xu, P., He, Y., \& Gong, G. (2013). PANDA: a pipeline toolbox for analyzing brain diffusion images. Front Hum Neurosci, 7, 42. doi:10.3389/fnhum.2013.00042

3. De Jager, P. L., Chibnik, L. B., Cui, J., Reischl, J., Lehr, S., Simon, K. C.,. .. Karlson, E. W. (2009). Integration of genetic risk factors into a clinical algorithm for multiple sclerosis susceptibility: a weighted genetic risk score. Lancet Neurol, 8(12), 1111-1119. doi:10.1016/s1474-4422(09)70275-3

4. de Rojas, I., Moreno-Grau, S., Tesi, N., Grenier-Boley, B., Andrade, V., Jansen, I. E.,. .. Ruiz, A. (2021). Common variants in Alzheimer's disease and risk stratification by polygenic risk scores. Nat Commun, 12(1), 3417. doi:10.1038/s41467-021-22491-8

5. Folstein, M. F., Folstein, S. E., \& McHugh, P. R. (1975). "Mini-mental state". A practical method for grading the cognitive state of patients for the clinician. J Psychiatr Res, 12(3), 189-198.

6. Hempel, R., Onopa, R., \& Convit, A. (2012). Type 2 diabetes affects hippocampus volume differentially in men and women. Diabetes Metab Res Rev, 28(1), 76-83. doi:10.1002/dmrr.1230

7. Jenkinson, M., Beckmann, C. F., Behrens, T. E., Woolrich, M. W., \& Smith, S. M. (2012). FSL. Neuroimage, 62(2), 782-790. doi:10.1016/j.neuroimage.2011.09.015

8. Li, C., Zhang, J., Qiu, M., Liu, K., Li, Y., Zuo, Z.,. .. Xiong, K. (2020). Alterations of Brain Structural Network Connectivity in Type 2 Diabetes Mellitus Patients With Mild Cognitive Impairment. Front Aging Neurosci, 12, 615048. doi:10.3389/fnagi.2020.615048 
9. McCrimmon, R. J., Ryan, C. M., \& Frier, B. M. (2012). Diabetes and cognitive dysfunction. Lancet, 379(9833), 2291-2299. doi:10.1016/s0140-6736(12)60360-2

10. Milne, N. T., Bucks, R. S., Davis, W. A., Davis, T. M. E., Pierson, R., Starkstein, S. E., \& Bruce, D. G. (2018). Hippocampal atrophy, asymmetry, and cognition in type 2 diabetes mellitus. Brain Behav, 8(1), e00741. doi:10.1002/brb3.741

11. Padilla-Martínez, F., Collin, F., Kwasniewski, M., \& Kretowski, A. (2020). Systematic Review of Polygenic Risk Scores for Type 1 and Type 2 Diabetes. Int J Mol Sci, 21(5). doi:10.3390/ijms21051703

12. Pedraza, O., Bowers, D., \& Gilmore, R. (2004). Asymmetry of the hippocampus and amygdala in MRI volumetric measurements of normal adults. J Int Neuropsychol Soc, 10(5), 664-678. doi: $10.1017 / \mathrm{s} 1355617704105080$

13. Preacher, K. J., \& Hayes, A. F. (2008). Asymptotic and resampling strategies for assessing and comparing indirect effects in multiple mediator models. Behav Res Methods, 40(3), 879-891. doi:10.3758/brm.40.3.879

14. Qi, D., Wang, A., Chen, Y., Chen, K., Zhang, S., Zhang, J.,. .. Zhang, Z. (2017). Default Mode Network Connectivity and Related White Matter Disruption in Type 2 Diabetes Mellitus Patients Concurrent with Amnestic Mild Cognitive Impairment. Curr Alzheimer Res, 14(11), 1238-1246. doi:10.2174/1567205014666170417113441

15. Riederer, P., Korczyn, A. D., Ali, S. S., Bajenaru, O., Choi, M. S., Chopp, M.,. .. Cukierman-Yaffe, T. (2017). The diabetic brain and cognition. J Neural Transm (Vienna), 124(11), 1431-1454. doi:10.1007/s00702-017-1763-2

16. Rosenberg, S. J., Ryan, J. J., \& Prifitera, A. (1984). Rey Auditory-Verbal Learning Test performance of patients with and without memory impairment. J Clin Psychol, 4O(3), 785-787.

17. Rubinov, M., \& Sporns, O. (2010). Complex network measures of brain connectivity: uses and interpretations. Neuroimage, 52(3), 1059-1069. doi:10.1016/j.neuroimage.2009.10.003

18. Sanjari Moghaddam, H., Ghazi Sherbaf, F., \& Aarabi, M. H. (2019). Brain microstructural abnormalities in type 2 diabetes mellitus: A systematic review of diffusion tensor imaging studies. Front Neuroendocrinol, 55, 100782. doi:10.1016/j.yfrne.2019.100782

19. Shin, M. S., Park, S. Y., Park, S. R., Seol, S. H., \& Kwon, J. S. (2006). Clinical and empirical applications of the Rey-Osterrieth Complex Figure Test. Nat Protoc, 1(2), 892-899. doi:10.1038/nprot.2006.115

20. Shu, N., Liang, Y., Li, H., Zhang, J., Li, X., Wang, L.,. .. Zhang, Z. (2012). Disrupted topological organization in white matter structural networks in amnestic mild cognitive impairment: relationship to subtype. Radiology, 265(2), 518-527. doi:10.1148/radiol.12112361

21. Spracklen, C. N., Horikoshi, M., Kim, Y. J., Lin, K., Bragg, F., Moon, S.,. . Sim, X. (2020). Identification of type 2 diabetes loci in 433,540 East Asian individuals. Nature, 582(7811), 240-245. doi:10.1038/s41586-020-2263-3

22. Sun, Q., Chen, G. Q., Wang, X. B., Yu, Y., Hu, Y. C., Yan, L. F.,. .. Cui, G. B. (2018). Alterations of White Matter Integrity and Hippocampal Functional Connectivity in Type 2 Diabetes Without Mild Cognitive 
Impairment. Front Neuroanat, 12, 21. doi:10.3389/fnana.2018.00021

23. van Bussel, F. C., Backes, W. H., van Veenendaal, T. M., Hofman, P. A., van Boxtel, M. P., Schram, M. T., .. Jansen, J. F. (2016). Functional Brain Networks Are Altered in Type 2 Diabetes and Prediabetes: Signs for Compensation of Cognitive Decrements? The Maastricht Study. Diabetes, 65(8), 24042413. doi:10.2337/db16-0128

24. Wimberley, T., Gasse, C., Meier, S. M., Agerbo, E., MacCabe, J. H., \& Horsdal, H. T. (2017). Polygenic Risk Score for Schizophrenia and Treatment-Resistant Schizophrenia. Schizophr Bull, 43(5), 10641069. doi:10.1093/schbul/sbx007

25. Xiong, Y., Chen, X., Zhao, X., Fan, Y., Zhang, Q., \& Zhu, W. (2020). Altered regional homogeneity and functional brain networks in Type 2 diabetes with and without mild cognitive impairment. Sci Rep, 10(1), 21254. doi:10.1038/s41598-020-76495-3

26. Zhang, Y., Zhang, X., Zhang, J., Liu, C., Yuan, Q., Yin, X.,. .. Wang, J. (2014). Gray matter volume abnormalities in type 2 diabetes mellitus with and without mild cognitive impairment. Neurosci Lett, 562, 1-6. doi:10.1016/j.neulet.2014.01.006

27. Zhou, H., Lu, W., Shi, Y., Bai, F., Chang, J., Yuan, Y.,. .. Zhang, Z. (2010). Impairments in cognition and resting-state connectivity of the hippocampus in elderly subjects with type 2 diabetes. Neurosci Lett, 473(1), 5-10. doi:10.1016/j.neulet.2009.12.057

28. Zung, W. W. (1965). A SELF-RATING DEPRESSION SCALE. Arch Gen Psychiatry, 12, 63-70.

29. Zung, W. W. (1971). A rating instrument for anxiety disorders. Psychosomatics, 12(6), 371-379. doi:10.1016/s0033-3182(71)71479-0

\section{Figures}

\section{Figure 1}

Correlations between the nodal properties of the right hippocampus and the PRS in the two groups. (a), The NGe of the right hippocampus was positively correlated with the PRS $(r=0.366, P<0.001)$ in the individuals with type 2 diabetes; (b), The NLp of the right hippocampus was negatively correlated with the PRS $(r=-0.366, P<0.001)$ in the individuals with type 2 diabetes; $(c)$, The $\mathrm{Nd}$ of the right hippocampus was positively correlated with the PRS $(r=0.320, P=0.001)$ in the individuals with type 2 diabetes. $N d$, nodal degree; NGe, nodal global efficiency; NLp, nodal shortest path length; R right.

\section{Figure 2}

Correlations between the nodal properties of the right hippocampus and the episodic memory tests in the individuals with type 2 diabetes. The NGe of the right hippocampus was positively correlated with the ROCF immediate recall $(r=0.252, P=0.012)(a)$, the ROCF delayed recall $(r=0.227, P=0.023)(b)$, and 
the AVLT long-term memory $(r=0.268, P=0.007)(c)$ in the individuals with type 2 diabetes. The NLp of the right hippocampus was negatively correlated with the ROCF immediate recall $(r=-0.239, P=0.016)$ (d), the ROCF delayed recall $(r=-0.219, P=0.028)(e)$, and the AVLT long-term memory $(r=-0.275, P=$ $0.006)$ (f) in the individuals with type 2 diabetes. AVLT, Auditory Verbal Learning Test; Nd, nodal degree; NGe, nodal global efficiency; NLp, nodal shortest path length; R right; ROCF, Rey-Osterrieth Complex Figure Test.

\section{Figure 3}

Mediation model of the PRS, the NGe of the right hippocampus, and episodic memory in individuals with type 2 diabetes. A significant direct effect was detected from the PRS to the NGe of the right hippocampus; and from the NGe of the right hippocampus to the episodic memory tests (ROCF immediate recall score, ROCF delayed recall score, AVLT long-term memory) in individuals with type 2 diabetes. A significant indirect effect was also found from the PRS to the episodic memory tests (ROCF immediate recall score, ROCF delayed recall score, AVLT long-term memory) mediated by the NGe of the right hippocampus. AVLT, Auditory Verbal Learning Test; Nd, nodal degree; NGe, nodal global efficiency; NLp, nodal shortest path length; R right; ROCF, Rey-Osterrieth Complex Figure Test.

\section{Figure 4}

Mediation model of the PRS, the NLp of the right hippocampus, and episodic memory in individuals with type 2 diabetes. A significant direct effect was detected from the PRS to the NLp of the right hippocampus; and from the NLp of the right hippocampus to the episodic memory tests (ROCF immediate recall score, ROCF delayed recall score, AVLT long-term memory) in individuals with type 2 diabetes. A significant indirect effect was also found from the PRS to the episodic memory tests (ROCF immediate recall score, ROCF delayed recall score, AVLT long-term memory) mediated by the NLp of the right hippocampus. AVLT, Auditory Verbal Learning Test; Nd, nodal degree; NGe, nodal global efficiency; NLp, nodal shortest path length; R right; ROCF, Rey-Osterrieth Complex Figure Test.

\section{Supplementary Files}

This is a list of supplementary files associated with this preprint. Click to download.

- Checklist.pdf 